\title{
Nonlinear eigenvalue problems for nonhomogeneous Leray-Lions operators
}

Mohamed Abdelwahed ${ }^{1}$ and Nejmeddine Chorfi ${ }^{\text {* }}$

\section{"Correspondence:}

nchorfi@ksu.edu.sa

'Department of Mathematics,

College of Sciences, King Saud

University, Riyadh, Saudi Arabia

\begin{abstract}
This paper deals with the mathematical analysis of a class of nonlinear eigenvalue problems driven by a nonhomogeneous differential operator. We are concerned both with the coercive and the noncoercive (and nonresonant) cases, which are in relationship with two associated Rayleigh quotients. The proof combines critical point theory arguments and the dual variational principle. The arguments developed in this paper can be extended to other classes of nonlinear eigenvalue problems with nonstandard growth.
\end{abstract}

MSC: Primary 35J60; secondary 47J10; 49R05; 58E05

Keywords: Nonhomogeneous differential operator; Nonlinear eigenvalue problem; Mountain pass theorem; Dual variational principle; Egorov theorem

\section{Introduction}

In a pioneering paper, Tolksdorf [1] studied Dirichlet problems involving the quasilinear elliptic second order differential operator

$$
\mathcal{A} u:=\operatorname{div}\left(a\left(|\nabla u|^{2}\right) \nabla u\right)
$$

where the potential $a:(0,+\infty) \rightarrow(0,+\infty)$ is of class $C^{1}$ and satisfies the following ellipticity and growth conditions of Leray-Lions type (cf. Leray and Lions [2]):

There are constants $\gamma, \Gamma>0, \kappa \in[0,1]$, and $p \in(1,+\infty)$ such that, for every $t>0$,

$$
\gamma t^{p-2} \leq a\left(t^{2}\right) \leq \Gamma(\kappa+t)^{p-2}
$$

and

$$
\left(\gamma-\frac{1}{2}\right) a(t) \leq t a^{\prime}(t) \leq \Gamma a(t)
$$

Thus, we can define the function $A:[0,+\infty) \rightarrow[0,+\infty)$ by $A(t)=\int_{0}^{t} a(s) d s$. Then $A$ is well defined for all $t \geq 0$ and is increasing and continuous on $[0,+\infty)$. Moreover, there exist

(c) The Author(s) 2020. This article is licensed under a Creative Commons Attribution 4.0 International License, which permits use, sharing, adaptation, distribution and reproduction in any medium or format, as long as you give appropriate credit to the original author(s) and the source, provide a link to the Creative Commons licence, and indicate if changes were made. The images or other third party material in this article are included in the article's Creative Commons licence, unless indicated otherwise in a credit line to the material. If material is not included in the article's Creative Commons licence and your intended use is not permitted by statutory regulation or exceeds the permitted use, you will need to obtain permission directly from the copyright holder. To view a copy of this licence, visit http://creativecommons.org/licenses/by/4.0/. 
constants $\gamma^{\prime}, \Gamma^{\prime}>0$, and $t_{0} \geq 0$ such that

$$
\gamma^{\prime} t^{p} \leq A\left(t^{2}\right) \leq \Gamma^{\prime} t^{p} \quad \text { for all } t \geq t_{0}
$$

Hence, in particular, $A$ satisfies the $\Delta_{2}$-condition near infinity (cf. Adams [3, p. 232]), that is,

$$
\limsup _{t \rightarrow+\infty} \frac{A(2 t)}{A(t)}<+\infty
$$

Conditions (1) and (2) also imply that

the mapping $\mathbb{R} \ni t \mapsto t a\left(t^{2}\right) \in \mathbb{R}$ is an odd increasing homeomorphism.

Indeed,

$$
\left(\operatorname{ta}\left(t^{2}\right)\right)^{\prime}=2 t^{2} a^{\prime}\left(t^{2}\right)+a\left(t^{2}\right) \geq(2 \gamma-1) a\left(t^{2}\right)+a\left(t^{2}\right)=2 \gamma a\left(t^{2}\right)>0
$$

It follows that the function

$$
t \mapsto A\left(t^{2}\right)=2 \int_{0}^{t} s a\left(s^{2}\right) d s
$$

is of class $C^{1}$ and convex on $\mathbb{R}$. This means that if $p=2$, then the potential $a(t)$ satisfies the hypotheses in the recent paper by Jeanjean and Rădulescu [4], which develops and extends previous contributions of Stuart [5].

By (2), we also observe that there exists $\eta>1$ such that

$$
t \frac{d}{d t} A\left(t^{2}\right) \geq \eta A\left(t^{2}\right) \quad \text { for all } t \geq 0
$$

To fix the ideas, we give examples of potentials $a$ satisfying (1) and (2) in the case $p=2$. Of course, related examples can be given for any $p>1$. For instance, if $a(t)=t^{(p-2) / 2}(t>0)$, we obtain the $p$-Laplace operator. We refer both to the Laplace operator (for $a(t) \equiv 1$ ) but also to combinations between the Laplace operator and the mean curvature operator, which is generated by

$$
a(t)= \begin{cases}\frac{1}{\sqrt{1+t}} & \text { if } t \in[0,1], \\ \alpha(t-2)^{2}+\beta & \text { if } t \in(1,2), \\ \beta & \text { if } t \in[2, \infty),\end{cases}
$$

where the real numbers $\alpha$ and $\beta$ are chosen to guarantee the smoothness of the potential a.

The analysis initiated by Tolksdorf [1] was extended by Omari and Zanolin [6] who studied the existence of infinitely many solutions for quasilinear elliptic problems driven by the operator $\operatorname{div}\left(a\left(|\nabla u|^{2}\right) \nabla u\right)$ and with oscillatory reaction. In particular, Omari and Zanolin introduced a notion of upper and lower solutions, which is adequate to the analysis of Dirichlet problems involving the quasilinear operator $\mathcal{A}$. Our purpose in the present paper 
is to use variational methods for the qualitative analysis of a class of nonlinear eigenvalue problems driven by the differential operator $\operatorname{div}\left(a\left(|\nabla u|^{2}\right) \nabla u\right)$.

The abstract tools used in this paper rely on some methods developed in the monographs [7-9].

\section{The main result}

Throughout this paper we assume that $\Omega \subset \mathbb{R}^{N}(N \geq 2)$ is a bounded domain with smooth boundary.

In order to describe the main contributions of the present paper, we recall the following classical results. Consider the problem

$$
\begin{cases}-\Delta u=\lambda u+|u|^{q-2} u & \text { in } \Omega, \\ u=0 & \text { on } \partial \Omega, \\ u>0 & \text { in } \Omega,\end{cases}
$$

where $1<q<2 N /(N-2)$ if $N \geq 3$ and $1<q<+\infty$ if $N=2$. Then, by the mountain pass theorem (see Ambrosetti and Rabinowitz [10]), problem (5) has a solution for all $\lambda<\lambda_{1}$, where $\lambda_{1}$ denotes the first eigenvalue of $(-\Delta)$ in $H_{0}^{1}(\Omega)$. We point out that the assumption $\lambda<\lambda_{1}$ is used to argue the existence of the "valley" condition in the Ambrosetti-Rabinowitz theorem. If $\lambda \geq \lambda_{1}$, then problem (5) does not have a mountain pass geometry. In this case we can deduce easily that problem (5) cannot have a positive solution. This follows easily by multiplication with $\varphi_{1}>0$ (the first eigenfunction of the Laplace operator) in (5) and integration over $\Omega$. We refer to Pucci and Rădulescu [11] for more details.

The aim of the present paper is to study the following nonlinear Dirichlet problem driven by a nonhomogeneous differential operator:

$$
\begin{cases}-\operatorname{div}\left(a\left(|\nabla u|^{2}\right) \nabla u\right)=\lambda a\left(u^{2}\right) u+|u|^{q-2} u & \text { in } \Omega, \\ u=0 & \text { on } \partial \Omega \\ u \neq \equiv 0 & \text { in } \Omega,\end{cases}
$$

where $\lambda$ is a real parameter and the potential $a$ satisfies hypotheses (1) and (2). We also assume that

$$
1<p<q<p^{*}:= \begin{cases}\frac{N p}{N-p} & \text { if } p<N \\ +\infty & \text { if } p \geq N\end{cases}
$$

In this paper we use standard notations and terminology. We denote by $W_{0}^{1, p}(\Omega)$ the Sobolev space equipped with the norm

$$
\left.\|u\|_{W_{0}^{1, p}(\Omega)}=\left(\int_{\Omega}|\nabla u|^{p}\right) d x\right)^{1 / p} .
$$

For simplicity we will often denote the above norm by $\|u\|$.

Define

$$
\Lambda:=\min \left\{\Lambda_{1}, \Lambda_{2}\right\},
$$


where

$$
\Lambda_{1}=\inf \left\{\int_{\Omega} A\left(|\nabla u|^{2}\right) d x ; u \in W_{0}^{1, p}(\Omega), \int_{\Omega} A\left(u^{2}\right) d x=1\right\}
$$

and

$$
\Lambda_{2}=\inf \left\{\int_{\Omega} a\left(|\nabla u|^{2}\right)|\nabla u|^{2} d x ; u \in W_{0}^{1, p}(\Omega), \int_{\Omega} a\left(u^{2}\right) u^{2} d x=1\right\} .
$$

The numbers $\Lambda_{1}$ and $\Lambda_{2}$ are associated with two different Rayleigh quotients. Since the problem is nonhomogeneous, these constrained minimization problems do not coincide.

We define a weak solution for problem (6) as a function $u \in W_{0}^{1, p}(\Omega) \backslash\{0\}$ satisfying for all $v \in W_{0}^{1, p}(\Omega)$

$$
\int_{\Omega} a\left(|\nabla u|^{2}\right) \nabla u \nabla v d x=\lambda \int_{\Omega} a\left(u^{2}\right) u v d x+\int_{\Omega}|u|^{q-2} u v d x .
$$

In this case, we say that $\lambda$ is an eigenvalue of problem (6) and the corresponding solution $u \in W_{0}^{1, p}(\Omega)$ is an eigenfunction corresponding to this eigenvalue. This definition is in accordance with the definition introduced by Fučik et al. [12, p. 117] in the case of nonlinear eigenvalue problems. Generally speaking, problem (6) can be understood as a nonlinear eigenvalue problem of the form

$$
S(u)=\lambda T(u), \quad \lambda \in \mathbb{R},
$$

where $S, T: X \rightarrow X^{*}$ ( $X$ is a Banach space) are nonlinear potential operators generated by the potentials $s, t: X \rightarrow \mathbb{R}$. In this framework, the element $\Lambda$ defined in (8) plays a crucial role for the existence of solutions to problem (6). In fact, $\Lambda$ is in close relationship with the principal eigenvalues of two nonlinear eigenvalue problems.

The main result of our paper establishes that in the "coercive" case $\lambda<\Lambda$, problem (6) admits at least one solution. In this case, we can also prove that this solution is positive. So, by the symmetry of (6), the problem has also a negative solution. However, in the "noncoercive" case corresponding to $\lambda \geq \Lambda$, we prove that (6) admits a solution but we do not have any sign information. This property is established in the "nonresonant" case, that is, if $\lambda$ is not an eigenvalue of a suitable pair of nonlinear operators.

Theorem 1 Assume that hypotheses (1), (2), and (7) are fulfilled.

(i) Then, for all $\lambda<\Lambda$, problem (6) has at least one positive solution.

(ii) Suppose that $\lambda \geq \Lambda$ and the operator $u \mapsto T u:=-\operatorname{div}\left(a\left(|\nabla u|^{2}\right) \nabla u\right)-\lambda a\left(u^{2}\right) u$ is bijective. Then problem (6) has at least one solution.

We point out that with similar arguments we can extend this result to the more general problem

$$
\begin{cases}-\operatorname{div}\left(a\left(|\nabla u|^{2}\right) \nabla u\right)=\lambda a\left(u^{2}\right) u+b\left(u^{2}\right) u & \text { in } \Omega, \\ u=0 & \text { on } \partial \Omega \\ u \neq \equiv 0 & \text { in } \Omega,\end{cases}
$$


where the potential $b:(0,+\infty) \rightarrow(0,+\infty)$ is of class $C^{1}$ and satisfies the following ellipticity and growth conditions of Leray-Lions type:

There exist constants $\gamma, \Gamma>0, \kappa \in[0,1]$, and $q \in\left(p, p^{*}\right)$ such that, for every $t>0$,

$$
\gamma t^{q-2} \leq b\left(t^{2}\right) \leq \Gamma(\kappa+t)^{q-2}
$$

and

$$
\left(\gamma-\frac{1}{2}\right) b(t) \leq t b^{\prime}(t) \leq \Gamma b(t)
$$

\section{The coercive case}

Let $\mathcal{J}: W_{0}^{1, p}(\Omega) \rightarrow \mathbb{R}$ be the variational functional defined by

$$
\mathcal{J}(u)=\frac{1}{2} \int_{\Omega}\left(A\left(|\nabla u|^{2}\right)-\lambda A\left(u^{2}\right)\right) d x-\frac{1}{q} \int_{\Omega}|u|^{q} d x .
$$

By hypothesis (7) and the Sobolev embedding theorem, $\mathcal{J}$ is well defined. Moreover, $\mathcal{J} \in$ $C^{1}\left(W_{0}^{1, p}(\Omega), \mathbb{R}\right)$ and its Gâteux directional derivative is given by

$$
\left\langle\mathcal{J}^{\prime}(u), v\right\rangle=\int_{\Omega}\left(a\left(|\nabla u|^{2}\right) \nabla u \nabla v-\lambda a\left(u^{2}\right) u v\right) d x-\int_{\Omega}|u|^{q-2} u v d x
$$

for any $u, v \in W_{0}^{1, p}(\Omega)$.

Since the problem has a variational structure, then solutions of problem (6) are critical points of the energy functional $\mathcal{J}$.

We define the truncation

$$
h(t)= \begin{cases}t^{q-1} & \text { if } t>0, \\ 0 & \text { if } t \geq 0,\end{cases}
$$

and we set $H(t):=\int_{0}^{t} h(s) d s$.

Consider the variational functional

$$
\mathcal{E}(u)=\frac{1}{2} \int_{\Omega}\left(A\left(|\nabla u|^{2}\right)-\lambda A\left(u^{2}\right)\right) d x-\int_{\Omega} H(u) d x .
$$

Then $\mathcal{E}$ is well defined, $\mathcal{E} \in C^{1}\left(W_{0}^{1, p}(\Omega)\right)$ and, for all $u, v \in W_{0}^{1, p}(\Omega)$,

$$
\left\langle\mathcal{E}^{\prime}(u), v\right\rangle=\int_{\Omega}\left[a\left(|\nabla u|^{2}\right) \nabla u \nabla v-\lambda a\left(u^{2}\right) u v\right] d x-\int_{\Omega} h(u) v d x .
$$

\subsection{Verification of the Palais-Smale condition}

Let $\left(u_{n}\right) \subset W_{0}^{1, p}(\Omega)$ be a Palais-Smale sequence of $\mathcal{E}$, that is,

$$
\mathcal{E}\left(u_{n}\right)=O(1) \quad \text { and } \quad\left\|\mathcal{E}^{\prime}\left(u_{n}\right)\right\|_{W^{-1, p^{\prime}}(\Omega)}=o(1) \quad \text { as } n \rightarrow \infty .
$$

It follows that

$$
\frac{1}{2} \int_{\Omega} A\left(\left|\nabla u_{n}\right|^{2}\right) d x-\frac{\lambda}{2} \int_{\Omega} A\left(u_{n}^{2}\right) d x-\int_{\Omega} H\left(u_{n}\right) d x=O(1)
$$


and, for all $v \in W_{0}^{1, p}(\Omega)$,

$$
\int_{\Omega} a\left(\left|\nabla u_{n}\right|^{2}\right) \nabla u_{n} \nabla v d x-\lambda \int_{\Omega} a\left(u_{n}^{2}\right) u_{n} v d x-\int_{\Omega} h\left(u_{n}\right) v d x=o(1)\|v\| .
$$

Taking $v=u_{n}$ in (11), we obtain

$$
\int_{\Omega} a\left(\left|\nabla u_{n}\right|^{2}\right) \mid \nabla u_{n}^{2} d x-\lambda \int_{\Omega} a\left(u_{n}^{2}\right) u_{n}^{2} d x-\int_{\Omega} h\left(u_{n}\right) u_{n} d x=o(1)\left\|u_{n}\right\| .
$$

But, for all $n \geq 1$,

$$
\int_{\Omega} h\left(u_{n}\right) u_{n} d x=q \int_{\Omega} H\left(u_{n}\right) d x
$$

Relations (10), (11), and (13) yield

$$
\begin{aligned}
(1-q) \int_{\Omega} H\left(u_{n}\right) d x= & \int_{\Omega}\left[\frac{1}{2} A\left(\left|\nabla u_{n}\right|^{2}\right)-a\left(\left|\nabla u_{n}\right|^{2}\right)\left|\nabla u_{n}\right|^{2}\right] d x \\
& -\lambda \int_{\Omega}\left[\frac{1}{2} A\left(u_{n}^{2}\right)-a\left(u_{n}^{2}\right) u_{n}^{2}\right] d x+O(1) \text { as } n \rightarrow \infty .
\end{aligned}
$$

But $A\left(t^{2}\right)=2 \int_{0}^{t} s a\left(s^{2}\right) d s$ and the mapping $(0,+\infty) \ni s \mapsto s a\left(s^{2}\right)$ is increasing. Therefore

$$
A\left(t^{2}\right) \leq 2 t^{2} a\left(t^{2}\right) \quad \text { for all } t \geq 0 \text {. }
$$

Claim. The sequence $\left(u_{n}\right) \subset W_{0}^{1, p}(\Omega)$ is bounded.

For this purpose, we first show that

$$
\text { the sequence }\left(u_{n}\right) \text { is bounded in } L^{q}(\Omega) \text {. }
$$

Assume first that $\lambda \leq 0$. Thus, from (14) and (15) we deduce that

$$
0 \leq(q-1) \int_{\Omega} H\left(u_{n}\right) d x \leq O(1) \quad \text { as } n \rightarrow \infty .
$$

hence $\left(u_{n}\right)$ is bounded in $L^{q}(\Omega)$.

If $\lambda>0$, relations (14) and (15) yield

$$
0 \leq(q-1) \int_{\Omega} H\left(u_{n}\right) d x \leq \lambda \int_{\Omega}\left[\frac{1}{2} A\left(u_{n}^{2}\right)-a\left(u_{n}^{2}\right) u_{n}^{2}\right] d x .
$$

Since $1<p<q$, relation (17) shows that $\left(u_{n}\right)$ is bounded in $L^{q}(\Omega)$.

By (8) and since $\lambda<\Lambda$, there exists $c_{0}>0$ such that, for all $u \in W_{0}^{1, p}(\Omega)$,

$$
\int_{\Omega} A\left(|\nabla u|^{2}\right) d x-\lambda \int_{\Omega} A\left(u^{2}\right) d x \geq c_{0} \int_{\Omega} A\left(|\nabla u|^{2}\right) d x .
$$

Returning to (10) and using (16) in combination with (18) and the hypothesis $\lambda<\Lambda$, we obtain the claim. 
Next, we prove that $\left(u_{n}\right) \subset W_{0}^{1, p}(\Omega)$ contains a strongly convergent subsequence. Relation (11) yields, for all $v \in W_{0}^{1, p}(\Omega)$,

$$
\int_{\Omega} a\left(\left|\nabla u_{n}\right|^{2}\right) \nabla u_{n} \nabla v d x=\int_{\Omega} \psi\left(u_{n}\right) v d x+o(1)\|v\| \quad \text { as } n \rightarrow \infty
$$

where

$$
\psi(w)=\lambda a\left(w^{2}\right) w+h(w) \quad \text { for all } w \in W_{0}^{1, p}(\Omega) .
$$

Obviously, $\psi$ is a continuous function.

Assume that $1<p<N$ (similar arguments work if $p \geq N$ ). It follows that

$$
|\psi(w)| \leq C\left(1+|w|^{(N p-N+p) /(N-p)}\right) \quad \text { for all } w \in W_{0}^{1, p}(\Omega)
$$

and

$$
\psi(w)=o\left(|w|^{N p /(N-p)}\right) \quad \text { as }|w| \rightarrow \infty .
$$

A crucial idea in the proof is to show that the sequence $\left\{\psi\left(u_{n}\right)\right\} \subset W^{-1, p^{\prime}}(\Omega)$ contains a strongly convergent subsequence. Indeed, in this case, relation (19) combined with the Sobolev embedding theorem implies that, up to a subsequence, $\left\{\psi\left(u_{n}\right)\right\}$ converges strongly in $\left(L^{N p /(N-p)}(\Omega)\right)^{*}=L^{N p /(N p-N+p)}(\Omega)$.

By our claim and the Rellich-Kondrachov embedding theorem, we can assume, up to a subsequence, that

$$
u_{n} \rightarrow u \quad \text { in } L^{N p /(N-p)}(\Omega)
$$

Fix $\delta>0$. Thus, by the Egorov theorem, there exists $\omega \subset \Omega$ such that $|\omega|<\delta$ and

$$
u_{n} \rightarrow u \quad \text { uniformly in } \Omega \backslash \omega \text {. }
$$

Fix $\eta>0$ small enough. Thus, to conclude the proof, it is enough to show that

$$
\int_{\omega}\left|\psi\left(u_{n}\right)-\psi(u)\right|^{N p /(N p-N+p)} d x \leq \eta \quad \text { for all } n \text { big enough. }
$$

Relation (20) implies that

$$
\int_{\omega}|\psi(u)|^{N p /(N p-N+p)} d x \leq C \int_{\omega}\left(1+|u|^{N p /(N-p)}\right) d x
$$

and the right-hand side can be made smaller than any positive constant if we choose $\delta>0$ small enough.

Next, by (21),

$$
\int_{\omega}\left|\psi\left(u_{n}\right)-\psi(u)\right|^{N p /(N p-N+p)} d x \leq \varepsilon \int_{\omega}\left|u_{n}-u\right|^{N p /(N-p)} d x+C_{\varepsilon}|\omega|,
$$


and the right-hand side can be made as small as we wish. This follows by combining our claim with the Sobolev embedding theorem.

We conclude that the energy functional $\mathcal{E}$ satisfies the Palais-Smale condition.

\subsection{Proof of Theorem 1(i) concluded}

We first prove that $\mathcal{E}$ satisfies the geometric hypotheses of the mountain pass theorem.

Fix $\lambda<\Lambda$. We have for all $u \in W_{0}^{1, p}(\Omega)$

$$
\begin{aligned}
\mathcal{E}(u) & \geq \frac{c_{0}}{2} \int_{\Omega} A\left(|\nabla u|^{2}\right) d x-\int_{\Omega} H(u) d x \\
& \geq \frac{c_{0}}{2} \int_{\Omega} A\left(|\nabla u|^{2}\right) d x-\frac{1}{q} \int_{\Omega}|u|^{q} d x,
\end{aligned}
$$

where $c_{0}$ is as in (18).

Next, by (3), we obtain for all $u \in W_{0}^{1, p}(\Omega)$

$$
\mathcal{E}(u) \geq \frac{c_{0} \gamma^{\prime}}{2} \int_{\Omega}|\nabla u|^{p} d x-\frac{1}{q} \int_{\Omega}|u|^{q} d x .
$$

Fix $\varepsilon>0$ small enough. Thus, by (7) and the Sobolev embedding theorem, there exists $r>0$ such that

$$
\mathcal{E}(u) \geq \varepsilon \quad \text { for all } u \in W_{0}^{1, p}(\Omega) \text { with }\|u\|=r .
$$

This establishes the existence of a "mountain" near the origin.

Next, we show the existence of a "valley" far from the origin.

Let $\varphi_{1}>0$ be the first eigenfunction of the Laplace operator, hence $\varphi_{1} \in W_{0}^{1, p}(\Omega)$. For all $t>0$, we have

$$
\begin{aligned}
\mathcal{E}\left(t \varphi_{1}\right)= & \frac{1}{2} \int_{\Omega}\left(A\left(t^{2}\left|\nabla \varphi_{1}\right|^{2}\right)-\lambda A\left(t^{2} \varphi_{1}^{2}\right)\right) d x-\frac{t^{q}}{q} \int_{\Omega} \varphi_{1}^{q} d x \\
\leq & \frac{1}{2} \int_{\left[t\left|\nabla \varphi_{1}\right| \geq t_{0}\right]} A\left(t^{2}\left|\nabla \varphi_{1}\right|^{2}\right) d x+\frac{1}{2} \int_{\left[t\left|\nabla \varphi_{1}\right|<t_{0}\right]} A\left(t^{2}\left|\nabla \varphi_{1}\right|^{2}\right) d x \\
& -\frac{\lambda}{2} \int_{\left[t \varphi_{1} \geq t_{0}\right]} A\left(t^{2} \varphi_{1}^{2}\right) d x-\frac{\lambda}{2} \int_{\left[t \varphi_{1}<t_{0}\right]} A\left(t^{2} \varphi_{1}^{2}\right) d x-\frac{t^{q}}{q} \int_{\Omega} \varphi_{1}^{q} d x,
\end{aligned}
$$

where $t_{0}$ is defined in (3).

Next, we evaluate the terms arising in (22). By (3) we have

$$
\frac{1}{2} \int_{\left[t\left|\nabla \varphi_{1}\right| \geq t_{0}\right]} A\left(t^{2}\left|\nabla \varphi_{1}\right|^{2}\right) d x \leq c_{1} t^{p}, \quad \text { where } c_{1}=\frac{\Gamma^{\prime}}{2} \int_{\Omega}\left|\nabla \varphi_{1}\right|^{p} d x>0,
$$

and

$$
-\frac{\lambda}{2} \int_{\left[t \varphi_{1} \geq t_{0}\right]} A\left(t^{2} \varphi_{1}^{2}\right) d x \leq c_{2} t^{p}, \quad \text { where } c_{2}=\frac{|\lambda| \Gamma^{\prime}}{2} \int_{\Omega} \varphi_{1}^{p} d x>0 .
$$

Recall that $A\left(t^{2}\right)=2 \int_{0}^{t} s a\left(s^{2}\right) d s$. Thus, by (1),

$$
A(t) \leq 2 p^{-1} \Gamma\left[(\kappa+t)^{p}-\kappa^{p}\right] .
$$


It follows that

$$
\begin{aligned}
\frac{1}{2} \int_{\left[t\left|\nabla \varphi_{1}\right|<t_{0}\right]} A\left(t^{2}\left|\nabla \varphi_{1}\right|^{2}\right) d x \leq & \frac{\Gamma}{p} \int_{\left[t\left|\nabla \varphi_{1}\right|<t_{0}\right]}\left(\kappa+t\left|\nabla \varphi_{1}\right|\right)^{p} d x-c_{4} \\
& \times \frac{\Gamma}{p} \int_{\Omega}\left(\kappa+t_{0}\right)^{p} d x-c_{4}=O(1) .
\end{aligned}
$$

Similarly, we deduce that

$$
-\frac{\lambda}{2} \int_{\left[t \varphi_{1}<t_{0}\right]} A\left(t^{2} \varphi_{1}^{2}\right) d x \leq O(1)
$$

Returning now to (22) we obtain

$$
\mathcal{E}\left(t \varphi_{1}\right) \leq\left(c_{1}+c_{2}\right) t^{p}-c_{3} t^{q}+O(1)
$$

where $c_{3}=q^{-1} \int_{\Omega} \varphi_{1}^{q} d x>0$. Using (7) we deduce that $\mathcal{E}\left(t \varphi_{1}\right)<0$ for $t$ large enough.

We have verified all the hypotheses of the mountain pass theorem. It follows that $\mathcal{E}$ has a nontrivial critical point $u$. Thus, for all $v \in W_{0}^{1, p}(\Omega)$,

$$
\int_{\Omega} a\left(|\nabla u|^{2}\right) \nabla u \nabla v d x=\lambda \int_{\Omega} a\left(u^{2}\right) u v d x+\int_{\Omega} h(u) v d x .
$$

Taking $v=u^{-}$as a test function, we obtain

$$
\int_{\Omega} a\left(|\nabla u|^{2}\right)\left|\nabla u^{-}\right|^{2} d x-\lambda \int_{\Omega} a\left(u^{2}\right)\left|u^{-}\right|^{2} d x=0 .
$$

Finally, by (8) and since $\lambda<\Lambda$, we conclude that $u^{-}=0$, hence $u \geq 0$. This means that $h(u)=u^{q-1}$, so $u$ is a solution of problem (6).

It remains to show that $u>0$ in $\Omega$. For this purpose, we observe that relations (4) and (7) imply that the hypotheses of the generalized maximum principle of Pucci and Serrin [13] are fulfilled. We conclude that $u>0$ in $\Omega$.

\section{The noncoercive case}

In this section we are concerned with the proof of Theorem 1(ii). The basic idea in this case is to use the dual variational principle of Clarke [14]. For more details, we refer to the seminal paper of Clarke [14], which is concerned with the dual action principle and its applications to the existence of periodic solutions to Hamilton's equations.

We introduce as a new unknown the function $w=|u|^{q-2} u$. It follows that

$$
u=|w|^{r-2} w \quad \text { with } r=\frac{q}{q-1}>1
$$

Since the operator $T$ is bijective, problem (6) can be rewritten as

$$
u=T^{-1}(w)
$$

or, equivalently,

$$
|w|^{r-2} w=T^{-1}(w)
$$


The energy functional associated with problem $(23)$ is $\mathcal{I}: L^{r}(\Omega) \rightarrow \mathbb{R}$ defined by

$$
\mathcal{I}(w)=\frac{1}{r} \int_{\Omega}|w|^{r} d x-\int_{\Omega} \mathcal{T}(w) d x,
$$

where $\mathcal{T}(t)=\int_{0}^{t} T^{-1}(u) d u$.

Since $T^{-1}$ maps $L^{r}(\Omega)$ into $W^{2, r}(\Omega) \subset L^{q}(\Omega)$, it follows that $\mathcal{I}$ is well defined and of class $C^{1}$ on $L^{r}(\Omega)$.

Next, we observe that $\mathcal{I}$ satisfies the hypotheses of the mountain pass theorem. In what concerns the geometric hypotheses of the energy functional, we observe that the growth of the first term of $\mathcal{I}$ around the origin is $r=q /(q-1)$, while the second term of $\mathcal{I}$ behaves like $p /(p-1)$ near zero. By hypothesis (7), we deduce that $r<p /(p-1)$, so the first term of $\mathcal{I}$ is dominating in a neighborhood of the origin, which implies the existence of a "mountain" near zero. Since the second term of $\mathcal{I}$ is the dominating one at infinity, we deduce the existence of a "valley" far from the origin.

Next, we show that $\mathcal{I}$ satisfies the Palais-Smale condition. For this purpose, we first prove that any Palais-Smale sequence is bounded and then that it is relatively compact.

Let $\left(w_{n}\right) \subset L^{r}(\Omega)$ be a Palais-Smale sequence of $\mathcal{I}$, that is,

$$
\mathcal{I}\left(w_{n}\right)=O(1) \quad \text { as } n \rightarrow \infty
$$

and

$$
\left\|\mathcal{I}^{\prime}\left(w_{n}\right)\right\|_{L^{q(\Omega)}} \rightarrow 0 \quad \text { as } n \rightarrow \infty .
$$

By (25) we deduce that

$$
\left|w_{n}\right|^{r-2} w_{n}-T^{-1}\left(w_{n}\right) \rightarrow 0 \quad \text { in } L^{q}(\Omega) .
$$

Multiplying relation (26) by $w_{n}$, integrating over $\Omega$, and comparing with (24) we deduce that the sequence $\left(w_{n}\right)$ is bounded in $L^{r}(\Omega)$. So, up to a subsequence,

$$
w_{n} \rightarrow w \text { in } L^{r}(\Omega) .
$$

Next, since the operator $\mathcal{T}: L^{r}(\Omega) \rightarrow L^{q}(\Omega)$ is compact and the space $W^{2, r}(\Omega)$ is compactly embedded into $L^{q}(\Omega)$, we deduce that $\mathcal{T}\left(w_{n}\right) \rightarrow \mathcal{T}(w)$ in $L^{q}(\Omega)$, hence $w_{n} \rightarrow w$ in $L^{r}(\Omega)$ as $n \rightarrow \infty$.

We conclude that $\mathcal{T}$ has a critical point $w$, which is a solution of problem (23). Moreover, this solution is nontrivial since $\mathcal{T}(w)>0$. This completes the proof of Theorem 1 .

\subsection{Final remarks}

(i) Analyzing the proof of Theorem 1 we observe that the conclusion still remains true if the right-hand side $f(u)$ of problem (6) satisfies the almost critical growth condition

$$
f(u)=o\left(u^{p^{*}-1}\right) \quad \text { as } u \rightarrow+\infty(\text { if } p<N) .
$$

We have already checked the validity of the Palais-Smale condition under this more general assumption. 
(ii) By hypothesis (7), the reaction of problem (6) has a $(p-1)$-superlinear growth. This is due to the term $|u|^{q-2} u(q>p)$. We do not know whether Theorem 1 holds if $|u|^{q-2} u$ is replaced with a smooth subcritical nonlinearity $g(u)$ satisfying the weaker condition

$$
\lim _{u \rightarrow+\infty} \frac{g(u)}{u^{p-1}}=+\infty
$$

(iii) We conjecture that the results established in Theorem 1 remain true for the problem

$$
\begin{cases}-\operatorname{div}\left(a\left(|\nabla u|^{2}\right) \nabla u\right)=\lambda a\left(u^{2}\right) u+|u|^{q-2} u+V(x) & \text { in } \Omega \\ u=0 & \text { on } \partial \Omega \\ u \neq 0 & \text { in } \Omega\end{cases}
$$

provided that $V$ is an indefinite potential such that $\|V\|_{L^{\infty}(\Omega)}$ is small enough.

(iv) We consider that a very interesting research direction concerns the study of problem (6) in the framework of variable exponents, that is, if the potential $a:(0,+\infty) \rightarrow(0,+\infty)$ is replaced by $a=a(x, t): \Omega \times(0,+\infty) \rightarrow(0,+\infty)$ such that the following hypotheses hold: there are functions $\gamma, \Gamma: \Omega \rightarrow(0, \infty), \kappa: \Omega \rightarrow[0,1]$, and $p: \Omega \rightarrow(1,+\infty)$ such that, for all $t>0$ and $x \in \Omega$,

$$
\gamma(x) t^{p(x)-2} \leq a\left(x, t^{2}\right) \leq \Gamma(x)(\kappa(x)+t)^{p(x)-2}
$$

and

$$
\left(\gamma(x)-\frac{1}{2}\right) a(x, t) \leq t a_{t}(x, t) \leq \Gamma(x) a(t) .
$$

We refer to the monograph by Rădulescu and Repovš [15] for advances in the variational analysis of nonlinear PDEs with variable exponent.

(v) Finally, we consider that new properties can be obtained in the framework of "doublephase" problems, that is, if the left-hand side of problem (6) is replaced by

$$
-\operatorname{div}\left(a\left(|\nabla u|^{2}\right) \nabla u\right)-\operatorname{div}\left(b\left(|\nabla u|^{2}\right) \nabla u\right),
$$

where the potentials $a$ and $b$ satisfy hypotheses like (1) and (2) for different exponents, say $p$ and $r$. We refer to the recent papers [16-23], which are concerned either with doublephase problems or with eigenvalue problems in isotropic or anisotropic settings. We consider that the differential operator studied in the present paper could be a source of inspiration for further developments, eventually in critical or supercritical cases.

\section{Appendix}

In the proof of Theorem 1 we have used the following version of the mountain pass theorem, see Ambrosetti and Rabinowitz [10].

Theorem 2 Let $X$ be a real Banach space and $J: X \rightarrow \mathbb{R}$ be a $C^{1}$-functional. Assume that $J$ satisfies the Palais-Smale condition and the following hypotheses:

(i) There exist positive constants $r$ and $c_{0}$ such that $J(u) \geq c_{0}$ for all $u \in X$ with $\|u\|=r$; 
(ii) $J(0)=0$ and there exists $v \in X$ such that $\|v\|>r$ and $J(v)<c_{0}$.

\title{
Then the functional $J$ has at least one critical point.
}

\author{
Acknowledgements \\ Researchers Supporting Project number (RSP-2019/153), King Saud University, Riyadh, Saudi Arabia
}

Funding

Not applicable.

Availability of data and materials

Not applicable.

Competing interests

The authors declare that they have no competing interests.

Authors' contributions

The authors declare that the study was realized in collaboration with equal responsibility. All authors read and approved the final manuscript.

\section{Publisher's Note}

Springer Nature remains neutral with regard to jurisdictional claims in published maps and institutional affiliations.

Received: 28 April 2020 Accepted: 3 July 2020 Published online: 16 July 2020

\section{References}

1. Tolksdorf, P.: On the Dirichlet problem for quasilinear equations. Commun. Partial Differ. Equ. 9, $773-817$ (1983)

2. Leray, J., Lions, J.-L.: Quelques résultats de višik sur des problèmes elliptiques non linéaires par les méthodes de Minty-Browder. Bull. Soc. Math. Fr. 93, 97-107 (1965)

3. Adams, R.A.: Sobolev Spaces. Academic Press, New York (1975)

4. Jeanjean, L., Rădulescu, V.D.: Nonhomogeneous quasilinear elliptic problems: linear and sublinear cases. arXiv:2002.06813

5. Stuart, C.A.: Two positive solutions of a quasilinear elliptic Dirichlet problem. Milan J. Math. 79, 327-341 (2011)

6. Omari, P., Zanolin, F.: Infinitely many solutions of a quasilinear elliptic problem with an oscillatory potential. Commun. Partial Differ. Equ. 21, 721-733 (1996)

7. Brezis, H.: Analyse Fonctionnelle: Théorie et Applications, Masson, Paris (1992)

8. Papageorgiou, N.S., Rădulescu, V.D., Repovš, D.D.: Nonlinear Analysis-Theory and Methods. Springer, Cham (2019)

9. Struwe, M.: Variational Methods: Applications to Nonlinear Partial Differential Equations and Hamiltonian Systems. Springer, Heidelberg (1996)

10. Ambrosetti, A., Rabinowitz, P.H.: Dual variational methods in critical point theory. J. Funct. Anal. 14, 349-381 (1973)

11. Pucci, P., Rădulescu, V.D.: The impact of the mountain pass theory in nonlinear analysis: a mathematical survey. Boll. Unione Mat. Ital. IX(3), 543-584 (2010)

12. Fučik, S., Nečas, J., Souček, J., Souček, V.: Spectral Analysis of Nonlinear Operators, vol. 346. Springer, Berlin (1973)

13. Pucci, P., Serrin, J.B.: The Maximum Principle, vol. 73. Birkhäuser, Basel (2007)

14. Clarke, F.: Solution périodique des équations hamiltoniennes. C. R. Acad. Sci. Paris Sér. A-B 287, $951-952$ (1978)

15. Rădulescu, V.D., Repovš, D.D.: Partial Differential Equations with Variable Exponents: Variational Methods and Qualitative Analysis. CRC Press, Boca Raton (2015)

16. Bahrouni, A., Rădulescu, V.D., Repovš, D.D.: Double phase transonic flow problems with variable growth: nonlinear patterns and stationary waves. Nonlinearity 32(7), 2481-2495 (2019)

17. Bandle, C., Wagner, A.: Shape optimization for an elliptic operator with infinitely many positive and negative eigenvalues. Adv. Nonlinear Anal. 7(1), 49-66 (2008)

18. Papageorgiou, N.S., Rădulescu, V.D., Repovš, D.D.: Positive solutions for nonlinear parametric singular Dirichlet problems. Bull. Math. Sci. 9(3), 11-21 (2019)

19. Papageorgiou, N.S., Rădulescu, V.D., Repovš, D.D.: Ground state and nodal solutions for a class of double phase problems. Z. Angew. Math. Phys. 71(1), 1-15 (2020)

20. Ragusa, M.A., Tachikawa, A.: Regularity for minimizers for functionals of double phase with variable exponents. Adv. Nonlinear Anal. 9(1), 710-728 (2020)

21. Rădulescu, V.D.: Isotropic and anisotropic double-phase problems: old and new. Opusc. Math. 39, 259-279 (2019)

22. Boureanu, B.B., Udrea, C.: No-flux boundary value problems with anisotropic variable exponents. Commun. Pure Appl. Anal. 14, 881-896 (2015)

23. Boureanu, B.B.: A new class of nonhomogeneous differential operator and applications to anisotropic systems. Complex Var. Elliptic Equ. 61, 712-730 (2016) 